\title{
A Case of Mature Natural Killer-Cell Neoplasm Manifesting Multiple Choroidal Lesions: Primary Intraocular Natural Killer-Cell Lymphoma
}

\author{
Yoshiaki Tagawa $^{a}$ Kenichi Namba $^{a}$ Reiki Ogasawara ${ }^{b}$ Hiromi Kannoc \\ Susumu Ishida ${ }^{a}$ \\ Departments of ${ }^{a}$ Ophthalmology and ${ }^{b}$ Hematology, Hokkaido University Graduate School \\ of Medicine, and 'Department of Surgical Pathology, Hokkaido University Hospital, \\ Sapporo, Japan
}

\section{Key Words}

Natural killer-cell neoplasm · Choroidal tumor · Primary intraocular lymphoma - Epstein-Barr virus $\cdot$ CD56

\begin{abstract}
Purpose: Natural killer (NK) cell neoplasm is a rare disease that follows an acute course and has a poor prognosis. It usually emerges from the nose and appears in the ocular tissue as a metastasis. Herein, we describe a case of NK-cell neoplasm in which the eye was considered to be the primary organ. Case: A 50-year-old female displayed bilateral anterior chamber cells, vitreous opacity, bullous retinal detachment, and multiple white choroidal mass lesions. Although malignant lymphoma or metastatic tumor was suspected, various systemic examinations failed to detect any positive results. A vitrectomy was performed OS; however, histocytological analyses from the vitreous sample showed no definite evidence of malignancy, and IL-10 concentration was low. Enlarged choroidal masses were fused together. Three weeks after the first visit, the patient suddenly developed an attack of fever, night sweat, and hepatic dysfunction, and 5 days later, she passed away due to multiple organ failure. Immunohistochemisty and in situ hybridization revealed the presence of atypical cells positive for CD3, CD56, and Epstein-Barr virus-encoded RNAs, resulting in the diagnosis of NK-cell neoplasm. With the characteristic clinical course, we concluded that this neoplasm was a primary intraocular NK-cell lymphoma. Conclusions: This is the first report to describe pri-
\end{abstract}

KARGER 125:s $\quad \begin{aligned} & \text { Kenichi Namba, MD, PhD } \\ & \text { Department of Ophthalmology } \\ & \text { Hokkaido University Graduate School of Medicine } \\ & \text { Kita-15, Nishi-7, Kita-ku, Sapporo 060-8638 (Japan) } \\ & \text { E-Mail knamba@med.hokudai.ac.jp }\end{aligned}$


Tagawa et al.: A Case of Mature Natural Killer-Cell Neoplasm Manifesting Multiple Choroidal Lesions: Primary Intraocular Natural Killer-Cell Lymphoma

mary intraocular NK-cell neoplasm. When we encounter atypical choroidal lesions, we should consider the possibility of NK-cell lymphoma, even though it is a rare disease.

\section{Introduction}

More than $90 \%$ of all ocular lymphoid neoplasms are B-cell lymphomas. Of the remainder of the cases, T-cell lymphoma and natural killer (NK)-cell neoplasm are rare, especially NK-cell neoplasm. According to the classification proposed by Suzuki et al. [1], two types of NK-cell neoplasm are recognized, namely extranodal NK-cell lymphoma and the nasal type (ENKL). Aggressive NK-cell lymphoma/leukemia (ANKL) has also been mentioned [2]. Among them, more than $70 \%$ of all NK-cell neoplasms are ENKL [1]. To the best of our knowledge, this is the first report of NK-cell neoplasm in which the primary lesion was the eye.

\section{Case Presentation}

A 50-year-old female became aware of the presence of a visual field defect OS and visited an ophthalmology clinic. Multiple white mass lesions were seen in both fundi, and she was referred to our hospital.

At the first visit, best-corrected visual acuity was $1.2 \mathrm{OD}$ and $0.5 \mathrm{OS}$. Slit-lamp and ophthalmoscopic examinations showed bilateral vitreous opacity graded $1+\mathrm{OD}, 2+\mathrm{OS}$, bullous serous retinal detachment, and multiple white choroidal mass lesions around the peripheral retina.

Systemic screening examinations were performed, but blood test results, particularly 5S-cysteinyldopa, soluble interleukin-2 receptor, and any other tumor markers, were negative. Contrast-enhanced body computed tomography, systemic positron emission tomography, contrast-enhanced cranial magnetic resonance imaging, and nasal endoscopy were performed; however, none of them displayed any abnormal findings, with the exception of contrast enhancement on the choroid by contrast-enhanced cranial magnetic resonance imaging.

Because vitreous opacity and serous retinal detachment were exacerbated rapidly in both eyes, a vitrectomy OS was performed on the fourth day in order to make a diagnosis. A cytological examination of the removed vitreous materials was performed using cell block preparation. At the time, cytopathologic evaluation revealed very few degenerated lymphoid cells present, but definite evidence of malignancy could not be pointed out. The IL- 6 count in the vitreous fluid was $5,600 \mathrm{pg} / \mathrm{ml}$ and that of IL-10 was $170 \mathrm{pg} / \mathrm{ml}$. A polymerase chain reaction (PCR) examination showed no detectable clonal rearrangement of the immunoglobulin heavy chain gene. Although we strongly suspected malignant lymphoma, we could not obtain the evidence to support the diagnosis.

With the administration of $60 \mathrm{mg}$ prednisolone, vitreous opacity decreased gradually; however, the number of choroidal lesions increased, each of which was extended and massively elevated (fig. 1). We proposed performing a choroidal biopsy; however, the patient did not agree with that course of action.

On the 25th day, the patient suddenly showed high fever up to $38^{\circ} \mathrm{C}$. Alanine aminotransferase had increased to $502 \mathrm{IU} / \mathrm{l}$, and lactate dehydrogenase had increased to 878 
Tagawa et al.: A Case of Mature Natural Killer-Cell Neoplasm Manifesting Multiple Choroidal Lesions: Primary Intraocular Natural Killer-Cell Lymphoma

IU/l. A bone marrow biopsy was performed; however, there was no evidence of abnormality. On the 29th day, the patient's symptoms included high fever of $40^{\circ} \mathrm{C}$ and shivering; lactate dehydrogenase was elevated to 10,391 IU/l. Hemophagocytic syndrome and multiple organ failure made us suspect malignant lymphoma, and cyclophosphamide/daunorubicine/vincristine/prednisolone treatment and steroid pulse therapy were administered. A bone marrow biopsy was performed again. On the 31st day, the patient passed away due to multiple organ failure.

After the patient had died, immunohistochemical examinations of the second bone marrow biopsy were performed, which revealed the existence of atypical cells that were positive for CD3, CD56, granzyme B, and in situ hybridization of Epstein-Barr virus-encoded small RNAs (EBER-ISH; fig. 2). As a result, we were able to diagnose this case as NK-cell neoplasm.

A vitreous sample obtained by vitrectomy was reviewed by additional immunostaining against CD56 and EBER-ISH. A few atypical cells showed positive for CD56, granzyme B, and EBER-ISH, although the number of these cells was too small to prove the diagnosis of malignancy (fig. 2). Therefore, we clinicopathologically diagnosed this case as primary intraocular NK-cell lymphoma that suddenly developed systemic dissemination resulting in death.

\section{Discussion}

NK-cell lymphoma accounts for less than $3 \%$ of all malignant lymphomas [2], and most are of the nasal type [1]. In the current case, nasal endoscopy failed to find any tumors in the nose. The second bone marrow biopsy detected the presence of atypical lymphocytes, whereas the first biopsy did not, indicating that the lymphoma had not yet invaded the bone marrow or was too small to be detected at the first biopsy. These results indicated that the NK-cell neoplasm emerged bilaterally in the eyes first and then disseminated into the entire body at a certain point in the clinical course. Accordingly, we conclude that this neoplasm was a primary intraocular NK-cell lymphoma.

Several cases of NK-cell lymphoma that developed in the eye and/or orbit have been reported; however, the primary tissue in which the lymphoma emerged was the nose [3]. A case of primary orbit, but not intraocular, NK-cell lymphoma was previously reported in China [4]. To the best of our knowledge, the current case report is the first to describe primary intraocular NK-cell lymphoma.

Immunohistochemical analysis revealed that atypical cells found in the bone marrow and vitreous were positive for CD3, CD56, and EBER-ISH. Therefore, these results indicate that the atypical cells found in this case were NK-cell lymphoma.

More than $90 \%$ of all intraocular lymphoma cases are B-cell lymphomas. For an intraocular lymphoma diagnosis, the IL-10/IL-6 ratio, the clonal rearrangement of the immunoglobulin heavy chain gene, and pathology are typically examined from the vitreous sample [5]. A diagnosis of malignant lymphoma could not be reached in the current case because the IGH gene rearrangement showed no monoclonality, the IL-10/IL-6 ratio was low, and CD20 was negative in the pathological examination. These tests are thought to be effective for detecting B-cell lymphoma, but not for T-cell lymphoma or NK-cell lymphoma. It must be understood that while a rare case of T- or NK-cell intraocular lymphoma might be found, most intraocular lymphomas are B-cell lymphomas. If the disease is suspected to be NK-cell lymphoma, additional immunohistochemical analysis, such as staining with CD56, EBER-ISH, etc., should be performed. It has been reported that EB virus was detected with PCR in the aqueous humor of a patient with NK-cell lymphoma that invaded into the anterior chamber 
Case Reports in

Ophthalmology

\begin{tabular}{l|l}
\hline Case Rep Ophthalmol 2015;6:380-384 \\
\hline DOI: 10.1159/000442018 & $\begin{array}{l}\text { ○ 2015 The Author(s). Published by S. Karger AG, Basel } \\
\text { www.karger.com/cop }\end{array}$ \\
\hline
\end{tabular}

Tagawa et al.: A Case of Mature Natural Killer-Cell Neoplasm Manifesting Multiple Choroidal Lesions: Primary Intraocular Natural Killer-Cell Lymphoma

[3]. A PCR analysis of EB virus with aqueous humor might be useful for supporting the diagnosis of intraocular NK-cell lymphoma.

\section{Statement of Ethics}

This case report has been approved by the Ethics Committee of Hokkaido University Hospital, Sapporo, Japan.

\section{Disclosure Statement}

The authors have no financial interests to disclose in this work.

\section{References}

-1 Suzuki R, Suzumiya J, Yamaguchi M, Nakamura S, Kameoka J, Kojima H, et al: Prognostic factors for mature natural killer (NK) cell neoplasms: aggressive NK cell leukemia and extranodal NK cell lymphoma, nasal type. Ann Oncol 2010;21:1032-1040.

-2 The World Health Organization classification of malignant lymphomas in Japan: incidence of recently recognized entities. Lymphoma Study Group of Japanese Pathologists. Pathol Int 2000;50:696-702.

3 Kase S, Namba K, Kitaichi N, Ohno S: Epstein-Barr virus infected cells in the aqueous humour originated from nasal NK/T cell lymphoma. Br J Ophthalmol 2006;90:244-245.

-4 Dai W, Zhong M, Shen W, Zou K, Bai CG: Natural killer T-cell lymphoma originating from the orbit. Chin Med J (Engl) 2012;125:1677-1680.

5 Kimura K, Usui Y, Goto H; Japanese Intraocular Lymphoma Study Group: Clinical features and diagnostic significance of the intraocular fluid of 217 patients with intraocular lymphoma. Jpn J Ophthalmol 2012;56:383-389.
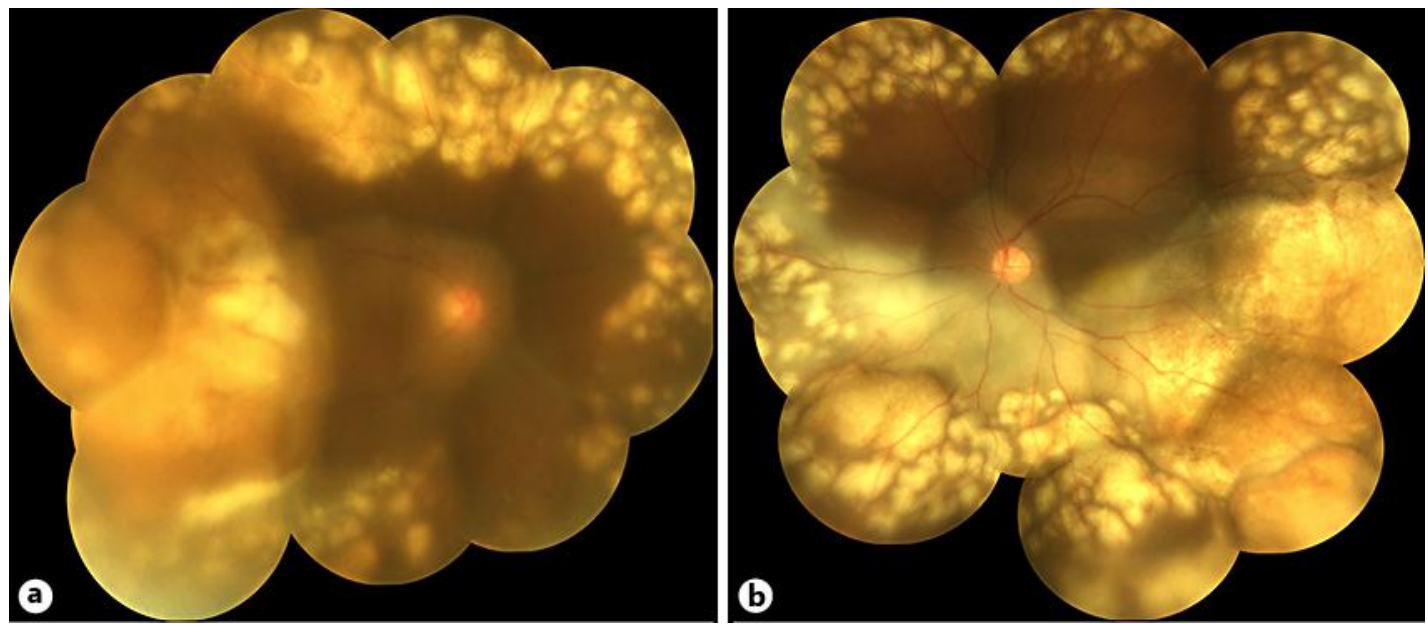

Fig. 1. The fundi 23 days after the patient's first visit. a Right eye, b left eye. Multiple white choroidal mass lesions were increased and extended abruptly. Choroidal protrusions were elevated bilaterally and massively in the temporal area. Around the inferior posterior pole of the left eye, the flat white lesion, which was suspected of having invaded lymphoma cells, extended into the subretinal space. 
Tagawa et al.: A Case of Mature Natural Killer-Cell Neoplasm Manifesting Multiple Choroidal Lesions: Primary Intraocular Natural Killer-Cell Lymphoma
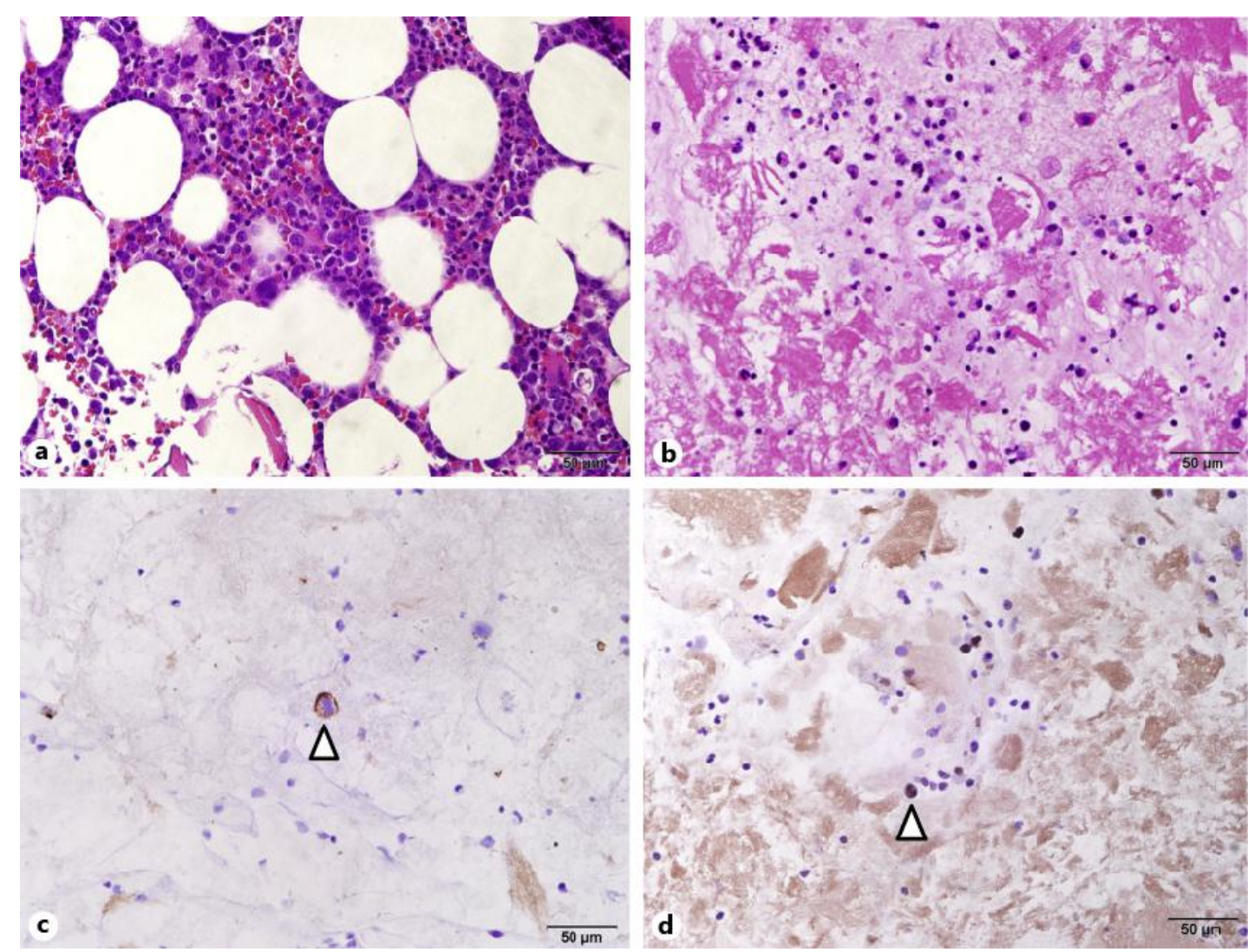

Fig. 2. Immunohistochemical analysis. In the second bone marrow biopsy, atypical cells were visualized with HE staining (a). In the vitreous sample cell block, a few atypical cells were visualized with HE staining (b) and identified as CD56 positive (white arrowhead; c) and EBER positive (white arrowhead; d). 